\title{
ASK Family Kinases Are Required for Optimal NLRP3 Inflammasome Priming
}

\author{
David E. Place, ${ }^{*}$ Parimal Samir, ${ }^{*}$ Rajendra Karki, ${ }^{*}$ Benoit Briard, ${ }^{*}$ Peter Vogel ${ }^{\dagger}$ and Thirumala-Devi Kanneganti*
}

From the Department of Immunology* and the Veterinary Pathology Core ${ }^{\dagger}$ St. Jude Children's Research Hospital, Memphis, Tennessee

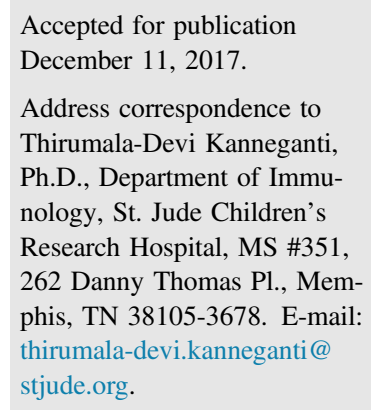

\begin{abstract}
Activation of the multimeric inflammasome complex leads to inflammatory responses to biotic and abiotic triggers. The inflammasome sensor, Nod-like receptor family pyrin domain containing 3 (NLRP3), is activated by a range of stimuli and is tightly regulated to restrict excessive inflammation. Because NLRP3 responds broadly to cellular insults and regulates cell death similar to the stress-activated apoptosis signal-regulating kinases 1 and 2 (ASK1/2), we hypothesized that ASK1/2 may regulate NLRP3 activity. Although essential for mediating NLRP3 inflammasome activation, ASK1/2 were not required for NLRC4 or absent in melanoma 2 inflammasome activation. ASK1/2 was required for NLRP3 up-regulation after lipopolysaccharide treatment in primary bone marrow-derived macrophages and lung fibroblasts as well as during infection with Burkholderia thailandensis and influenza virus. Consistent with reduced NLRP3 expression in response to $B$. thailandensis, caspase- 1 cleavage and cell death were reduced in infected bone marrow-derived macrophages, and mice lacking ASK1/2 were resistant to Burkholderia intranasal infection. Single knockouts of either ASK1 or ASK2 showed a partial role for both ASK1 and ASK2 in NLRP3 up-regulation in response to lipopolysaccharide or $B$. thailandensis, but ASK2 was required primarily to mediate lethal pathology during intranasal infection in vivo. Our findings identify the ASK1/2 complex as a regulator of NLRP3 activation and highlight a larger role for ASK2 in lung infection during $B$. thailandensis infection. (Am J Pathol 2018, 188: 1021-1030; https://doi.org/10.1016/j.ajpath.2017.12.006)
\end{abstract}

Mitogen-activated protein kinases (MAPKs) are Ser/Thr kinases that are involved in relaying extracellular signals to regulate survival, apoptosis, metabolism, inflammation, and many other cellular processes. ${ }^{1}$ Conventional MAPKs include the p38 isoforms, c-Jun amino (N)-terminal kinases $1 / 2 / 3$ (JNK1/2/3), and the extracellular signal-regulated kinases 1/2. These mitogen-activated protein kinase (MAPK)s are regulated by a series of upstream kinases (MAP2K and MAP3Ks) that also are evolutionarily conserved Ser/Thr kinases. ${ }^{2}$ As downstream targets of proinflammatory Toll-like receptor 4 (TLR4), MAP3Ks, including transforming growth factor- $\beta$-activated kinase 1 (alias MAP3K7) and apoptosis-signal regulating kinase 1 (ASK1, alias MAP3K5), phosphorylate downstream MKK4/7 and MKK3/6, followed by JNK and p38 MAPK activation. $^{2}$ Although transforming growth factor$\beta$-activated kinase 1 once was considered essential to activate JNK and p38 in vitro downstream of TLR activation, recent studies have suggested that this is not universally true in myeloid cells. ${ }^{3}$ Indeed, ASK1 also regulates activation of JNK and p38 downstream of TLRs, emphasizing the importance of understanding how each of these MAP3Ks regulates innate immunity.

The ASK family of MAP3Ks, ASK1, ASK2, and ASK3 (encoded by Map3k5, Map3k6, and Map3k15, respectively) are related by sequence homology and interact to regulate the activity of one another. ${ }^{4-6}$ ASK1 is the most studied family member and is an upstream kinase of the JNK and p38 pathways. ${ }^{7}$ Diverse stimuli lead to ASK1 activation including TLR signaling, reactive oxygen species, cytokines, and endoplasmic reticulum stress, and activation is inhibited by thioredoxin and 14-3-3 or promoted by TNF receptor associated factor $2 / 6.8,9$ ASK1 drives apoptotic cell death in response to tumor necrosis factor and $\mathrm{H}_{2} \mathrm{O}_{2}$, which

Supported by NIH grants AI101935, AI124346, AR056296, and CA163507 (all to T.-D.K.) and the American Lebanese Syrian Associated Charities (T.-D.K.). The St. Jude Children's Research Hospital Cell and Tissue Imaging Center is supported by St. Jude Children's Research Hospital and National Cancer Institute grant P30 CA021765-35.

Disclosures: None declared. 
can be regulated by ASK2 in certain circumstances, but its role in other forms of regulated cell death are not well studied. ${ }^{6,7,10,11}$ ASK1 and ASK2 also are important in regulating inflammation and cell death during influenza infection, but their role in bacterial infection models is not known, despite the well-established role of ASK1 in the TLR4 signaling pathway. ${ }^{12-14}$

The inflammasome is a multimeric protein complex that activates caspase- 1 and leads to an inflammatory cell death termed pyroptosis. ${ }^{15,16}$ The assembly of the inflammasome also involves an adaptor protein ASC [apoptosis-associated speck-like protein containing a caspase activation and recruitment domains (CARD)] containing a CARD and pyrin domain that facilitates the oligomerization of the various sensor proteins Nod-like receptor family pyrin domain containing 3 (NLRP3), NLR family CARD domain containing 4 (NLRC4), and absent in melanoma 2 (AIM2). ${ }^{16}$ NLRP3 is the most well-studied inflammasome sensor and NLRP3mediated inflammasome activation is downstream of a multitude of triggers that are reminiscent of the stimuli activating ASK1, including infectious agents such as influenza virus or bacteria. ${ }^{17,18} \mathrm{We}$ therefore hypothesized that the MAP3Ks ASK1 and ASK2 may regulate NLRP3 inflammasome activation in response to intracellular bacterial infection.

ASK1 and ASK2 contribute to the activation of the NLRP3 inflammasome but not the NLRC4 or AIM2 inflammasomes. ASK1 and ASK2 are required to enhance expression of NLRP3 in response to TLR4 stimulation by lipopolysaccharide (LPS), influenza virus, and Burkholderia thailandensis. ASK1/2-dependent NLRP3 inflammasome priming also is required for caspase-1 activation during $B$. thailandensis infection of bone marrow-derived macrophages (BMDMs) and causes lethal inflammation in an intranasal infection model. ASK1 and ASK2 both contribute partially to the NLRP3 priming in BMDMs treated with LPS or B. thailandensis, but in the intranasal model ASK2 predominantly drives lethal inflammation during infection, consistent with its higher expression in the lung. Together, these data show that in addition to its well-known role in apoptosis, the ASK1/2 signaling complex contributes to pyroptotic cell death by regulating the NLRP3 inflammasome.

\section{Materials and Methods}

\section{Mice}

Wild-type (WT) (C57BL6/J), Askl ${ }^{-/-}\left(M^{-1} 3 k 5^{\text {tm1Hijo }}\right){ }^{7}$

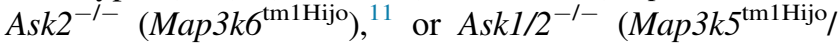
Map $\left.3 k 6^{\mathrm{tm} 1 \mathrm{Hijo}}\right)$ mice were obtained from the RIKEN BioResources Center (Tsukuba, Japan) and $\mathrm{Nlrp}^{-/-}, \mathrm{Aim}^{-{ }^{-1}}$,

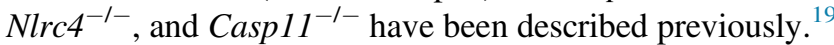
Male and female mice between 6 and 8 weeks old were used in this study. Mice were bred at St. Jude Children's Research Hospital and studies were conducted under protocols approved by St. Jude Children's Research Hospital on the Use and Care of Animals.

\section{Bone Marrow-Derived Macrophages}

Primary BMDMs were grown for 6 days in Dulbecco's modified Eagle medium (DMEM; 11995073; ThermoFisher Scientific, Waltham, MA) supplemented with $10 \%$ fetal bovine serum (FBS; TMS-013-B; Millipore, Billerica, MA; or S1620; BioWest, Riverside, MO), 30\% L929 conditioned media, and 1\% penicillin and streptomycin (15070-063; ThermoFisher Scientific). BMDMs were seeded in antibiotic-free media at a concentration of $1 \times 10^{6}$ cells onto 12 -well plates and incubated overnight. Cells then were washed and cultured in antibiotic-free DMEM with $10 \%$ FBS before stimulations or infections.

\section{In Vitro Isolation and Culture of Lung Fibroblasts}

Lung fibroblasts were prepared from the mouse lungs as described previously. ${ }^{20}$ Briefly, the lungs were harvested, cut into small pieces, and suspended in trypsin lysis buffer 0.25\% (25200-056; ThermoFisher Scientific) for 30 minutes at $37^{\circ} \mathrm{C}$ under agitation. Lung fibroblast medium (DMEM, $10 \%$ FBS, $1 \%$ penicillin-streptomycin, $1 \%$ nonessential amino acid, $1 \%$ sodium pyruvate, $1 \%$ HEPES buffer, $1 \%$ L-glutamine, and $0.25 \% \beta$-mercaptoethanol) was added to the suspension. The cell suspensions were centrifuged at $300 \times g$ for 5 minutes and pellets were resuspended in lung fibroblast medium and then cultured on tissue-culturetreated dishes. Lung fibroblasts $\left(2 \times 10^{5}\right)$ were seeded onto 12-well cell culture plates in DMEM supplemented with $10 \%$ FBS and $1 \%$ penicillin-streptomycin and stimulated as described for BMDMs.

\section{Bacterial Culture}

B. thailandensis strain E264 provided by Dr. Joseph Mougous (University of Washington, Seattle, WA) and Salmonella enterica Typhimurium SL1344 were cultured in Luria Bertani broth (3002-031; MP Biomedicals, Santa Ana, CA) overnight and subcultured into fresh Luria Bertani media for 3 hours at $37^{\circ} \mathrm{C}$ to generate $\log$-phase grown bacteria.

\section{Mouse Infections}

B. thailandensis was grown as described above (Bacterial Culture). For survival experiments, quantified frozen aliquots of B. thailandensis were diluted in phosphate-buffered saline before infection to inoculate at $5 \times 10^{5}$ bacteria per mouse. Mice were anesthetized with isoflurane and administered B. thailandensis in a $50-\mu \mathrm{L}$ phosphate-buffered saline suspension to the nares. After 2 days, lungs and spleens were harvested for histology or colony forming unit analysis. Colony forming units were determined by homogenizing tissue in phosphate-buffered saline with metal beads for 2 minutes using the Qiagen TissueLyser II apparatus (Qiagen, Venlo, Netherlands) and plating on Luria Bertani agar plates incubated overnight. 
Bone Marrow-Derived Macrophage Stimulations

BMDMs were differentiated as described above (Bone Marrow-Derived Macrophages). Before stimulation, cells were rinsed with phosphate-buffered saline and replaced with fresh antibiotic-free DMEM containing 10\% FBS. For inflammasome activation, the following conditions were used to stimulate BMDMs: ultrapure LPS from Salmonella minnesota R595 (tlrl-smlps; InvivoGen, San Diego, CA, at $50 \mathrm{ng} / \mathrm{mL}$ for 3 hours or transfected at $1 \mu \mathrm{g} / \mathrm{mL}$ for 20 hours), ATP (10127531001; Roche, Basel, Switzerland $5 \mathrm{mmol} / \mathrm{L}$ for 30 minutes), S. enterica subspecies Typhimurium (multiplicity of infection, 1 for 4 hours), poly dA:dT [poly(deoxyadenylicdeoxythymidylic) acid sodium salt; tlrl-patn; InvivoGen, transfected at $2.5 \mu \mathrm{g} / \mathrm{mL}$ for 4 hours], influenza A/Puerto Rico/ 8/34 virus PR8 [influenza A virus strain A/Puerto Rico/8/1934 H1N1; multiplicity of infection, 25 in FBS/L-glutamine/sodium pyruvate-free DMEM (D5671; Sigma, St. Louis, MO) for 2 hours, then addition of FBS to $10 \%$ until 20 hours], and B. thailandensis (multiplicity of infection, 10 for 20 hours). For transfection experiments, LPS and DNA were mixed with 0.3 $\mu \mathrm{L}$ Xfect polymer in Xfect reaction buffer (631318; Clontech Laboratories, Mountain View, CA) and incubated for $10 \mathrm{mi}-$ nutes before being added to BMDMs in Opti-MEM (31985070; ThermoFisher Scientific). B. thailandensis was pelleted onto cells at $300 \times g$ for 5 minutes, washed after 1 hour, incubated with DMEM (1000 $\mu \mathrm{g} / \mathrm{mL}$ kanamycin) for 1 hour, washed, and finally incubated in DMEM $(250 \mu \mathrm{g} / \mathrm{mL}$ kanamycin) for the remainder of the experiment.

\section{Immunoblotting Analysis}

For measuring caspase-1 cleavage, BMDMs and supernatant were collected together in buffer containing NP-40, dithiothreitol, protease inhibitor, and Laemmli sample buffer. For signaling blots, supernatant was removed and cells were lyzed in RIPA buffer containing protease and phosphatase inhibitors and Laemlli buffer. Proteins were separated via SDS-PAGE, with $6 \%$ to $12 \%$ polyacrylamide gels and polyvinylidene difluoride membranes (IPVH00010; Millipore), and blocked with 5\% skim milk. Primary antibodies against caspase-1 (AG-20B-0042; Adipogen, San Diego, CA), NLRP3 (AG-20B-00140C100; Adipogen), IL-1 $\beta$ (D4T2D; Cell Signaling Technologies, Danvers, MA), and $\beta$-actin (\#8457; Cell Signaling Technologies) were incubated overnight at $4^{\circ} \mathrm{C}$ followed by appropriate secondary antibodies conjugated with horseradish peroxidase incubated for 1 hour at room temperature (Jackson ImmunoResearch, West Grove, PA). Membranes were visualized using Luminata Forte Chemiluminescence substrate (WBLUF0500; Millipore).

\section{RT-PCR Analysis}

RNA was isolated using TRIzol (15596026; ThermoFisher Scientific) and converted into cDNA using the High-Capacity
cDNA Reverse Transcription kit (4369914; Applied Biosystems, Foster City, CA) according to the manufacturer's protocols. Real-time quantitative PCR was performed on an ABI 7500 real-time PCR machine (Applied Biosystems) with $2 \times$ SYBR Green (4368706; Applied Biosystems). Primer sequences for NLRP3 (forward: 5'-TGCAGAAGACTGACGTCTCC- $3^{\prime}$ and reverse: 5'-CGTACAGGCAGTAGAACAGTTC- $3^{\prime}$ ) and IL- $1 \beta$ (forward: 5'-GACCTTCCAGGATGAGGACA- $3^{\prime}$ and reverse: $5^{\prime}$-AGCTCATATGGGTCCGACAG-3') were used.

\section{Cytokine Analysis}

Cytokine levels were determined using an IL-18 enzymelinked immunosorbent assay (BMS618/3TEN; ThermoFisher Scientific) or multiplex enzyme-linked immunosorbent assay (MCYTOMAG-70K; Millipore) according to the manufacturer's instructions.

\section{Quantification and Statistical Analysis}

GraphPad Prism 6.0 software (La Jolla, CA) was used for data analysis. Data are shown as means \pm SEM. Statistical significance was determined by the Mann-Whitney test for two groups or one-way analysis of variance (with the Dunnett multiple comparison tests) for three or more groups. Survival curves were compared using the log-rank test. $P<0.05$ was considered statistically significant. Data from independent experiments were pooled where appropriate.

\section{Results}

\section{ASK1/2 Are Required for NLRP3 Inflammasome Activation}

Previous work on the ASK1/2 complex focused primarily on the role of downstream signaling on regulating apoptosis in cells exposed to cytokine, endoplasmic reticulum, or infectious or oxidative stressors. ${ }^{21}$ To examine the role of these kinases in inflammasome activation, primary BMDMs were subjected to activators of the canonical NLRP3, NLRC4, and AIM2 inflammasomes, and the noncanonical caspase-11/ NLRP3 inflammasome. The canonical NLRP3 inflammasome is activated by treatment with LPS for 3 hours followed by ATP. ${ }^{22,23}$ Upon priming of BMDMs with LPS followed by 30 minutes of ATP treatment, Askl/2 ${ }^{-/-}$macrophages showed reduced caspase- 1 cleavage and release of IL- $1 \beta$ and lactate dehydrogenase compared with WT (Figure 1, A and B). This suggests that ASK1/2 signaling is required for potentiation of NLRP3 inflammasome activation.

The NLRC4 inflammasome is activated by ligand binding to NLR family apoptosis inhibitory proteins, with bacterial type III secretion system needle proteins binding NLR family apoptosis inhibitory protein 1 , type III secretion system rod protein binding NLR family apoptosis inhibitory protein 2, and flagellin binding NLR family apoptosis 

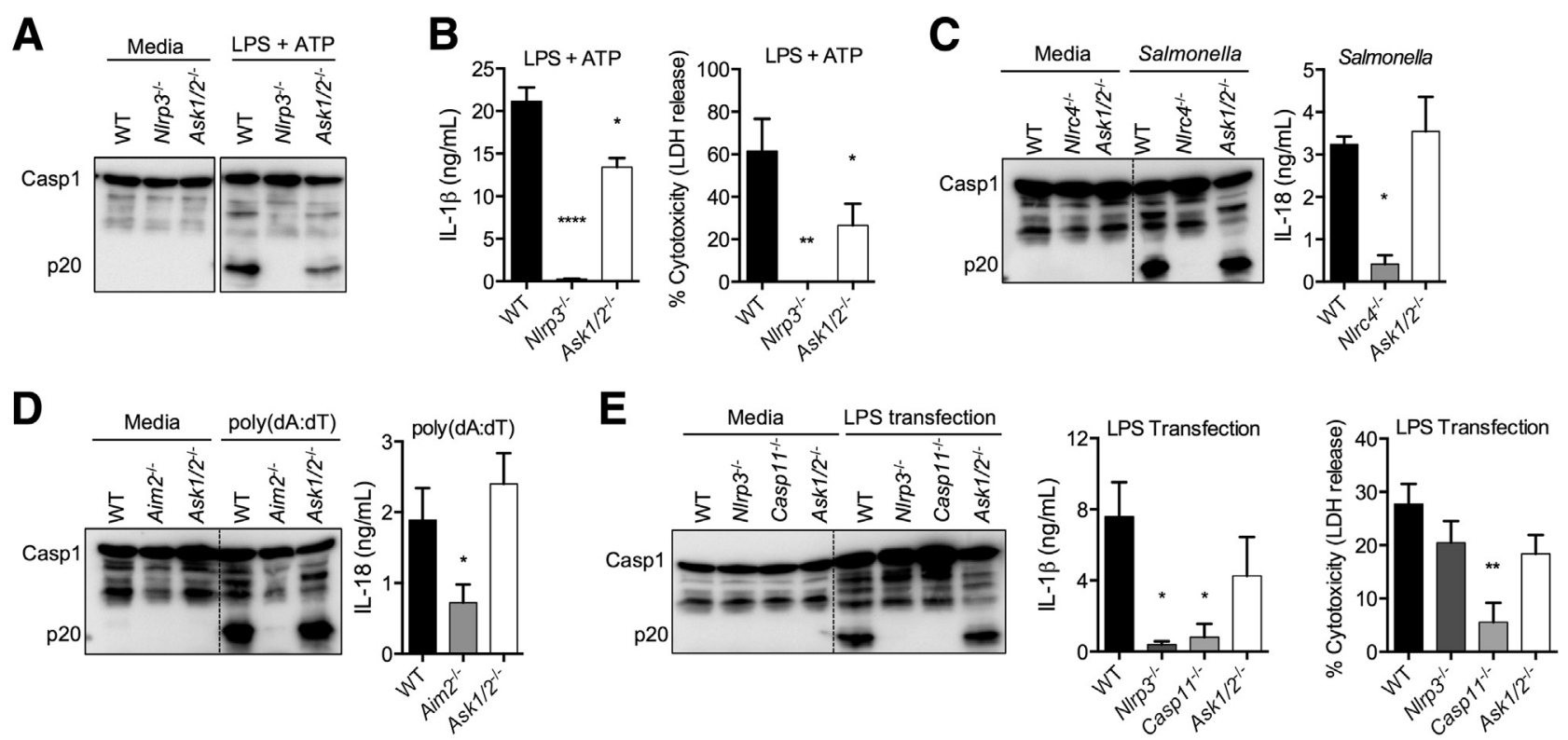

Figure 1 Apoptosis-signal regulating kinases 1/2 (ASK1/2) are required for activation of the Nod-like receptor family pyrin domain containing 3 (NLRP3) inflammasome in bone marrow-derived macrophages (BMDMs). A: Immunoblot analysis for caspase-1 cleavage (full-length Casp1 and Casp1 p20) in WT or knockout BMDMs after $50 \mathrm{ng} / \mathrm{mL}$ lipopolysaccharide (LPS) and $5 \mathrm{mmol} / \mathrm{L}$ ATP stimulation (same blot was cut to remove lanes). B: Supernatants were collected and release of lactate dehydrogenase (LDH) and IL-1 $\beta$ was performed. C-E: BMDMs were infected with $S$. enterica subspecies Typhimurium (multiplicity of infection, 1) (C), transfected with poly (dA:dT) (D), or transfected with LPS (E), and caspase-1 cleavage was measured by immunoblot, and IL-18 or IL-1 $\beta$ release into the supernatant was measured by enzyme-linked immunosorbent assay. Data are representative from at least three independent experiments with significance determined by one-way

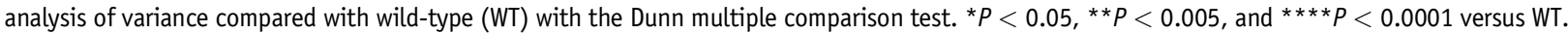

inhibitory proteins $5 / 6 .{ }^{24}$ WT and Ask1/2 ${ }^{-1-}$ BMDMs were infected with Salmonella and no defect was observed in NLRC4-dependent inflammasome activation or IL-18 release, suggesting ASK1/2 does not regulate the NLRC4 inflammasome (Figure 1C).

Recognition of cytoplasmic double-stranded DNA is mediated by the AIM2 inflammasome. ${ }^{25-28}$ BMDMs were transfected with poly(dA:dT), and no role for ASK1/2 signaling was found in the activation of the AIM2 inflammasome (Figure 1D). These data show that the AIM2 inflammasome does not require ASK1/2 signaling for activation.

In addition to the canonical NLRP3 inflammasome, noncanonical caspase-11/NLRP3 inflammasome activation occurs after intracellular LPS recognition by caspase-11 and downstream cleavage of gasdermin D, which through an unknown mechanism leads to NLRP3-dependent caspase-1 cleavage. ${ }^{29,30}$ Overexpression of ASK1 has been shown to induce the expression of caspase-11, and the noncanonical inflammasome also is primed by toll-like receptor adaptor molecule 1-dependent interferon up-regulation of caspase$11 .^{31-33}$ Given the role of ASK1/2-dependent signaling in influenza and poly(I:C)-mediated interferon production, ${ }^{13}$ BMDMs were transfected with LPS to determine whether ASK1/2-mediated signaling was required for the regulation of the caspase-11/NLRP3 noncanonical inflammasome. No role for ASK1/2-dependent signaling was observed in cleavage of caspase-1 or release of inflammasome-dependent cytokines after LPS transfection (Figure 1E), suggesting that intracellular
LPS sensing by caspase-11 does not require ASK1/2 activity. No defects were observed in cell death after LPS transfection, which occurs upstream of NLRP3 inflammasome activation (Figure 1E). Together, these data suggest the ASK1/2 complex regulates NLRP3 activation for acute time points after LPS stimulation, as in the case of LPS and ATP stimulation, but is not required during the extended LPS treatment used for intracellular LPS detection by caspase-11 and downstream gasdermin D and NLRP3-dependent caspase-1 cleavage.

\section{ASK1/2 Are Required for Up-Regulation of NLRP3 and} IL-1 $\beta$

The NLRP3 inflammasome is regulated by both a priming step consisting of transcriptional up-regulation and post-translational licensing followed by a second activation step that leads to the oligomerization of NLRP3 with ASC and caspase-1 to form a catalytically active inflammasome complex. ${ }^{34}$ To determine whether the NLRP3 priming step was regulated by ASK1/2 signaling, BMDMs were primed with LPS to induce the expression of NLRP3 and IL- $1 \beta$ and it was found that ASK1/2 are required for transcriptional and translational up-regulation of both NLRP3 and IL-1 $\beta$ (Figure 2, A and B). LPS-dependent up-regulation of NLRP3 in primary lung fibroblasts also required ASK1/2 signaling (Figure 2C). NLRP3 expression also was reduced in BMDMs infected with the Gram-negative intracellular bacterium B. thailandensis and mouse-adapted influenza A virus 
A

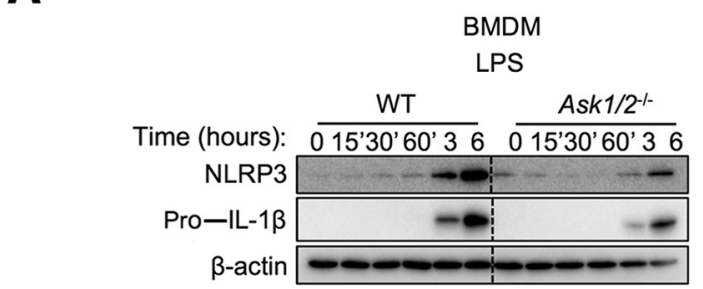

C

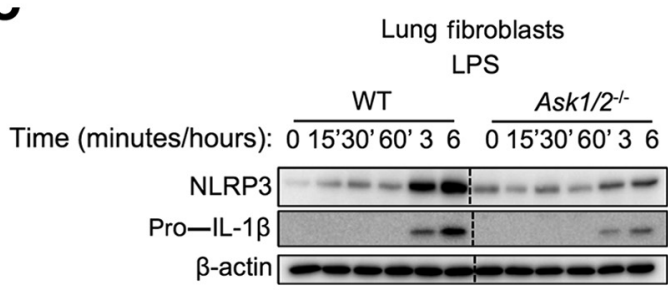

B

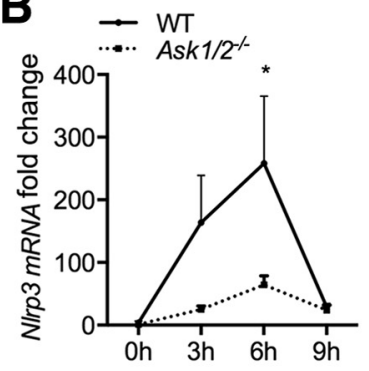

D

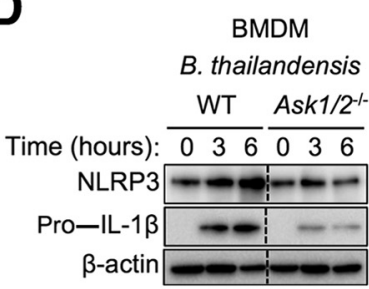

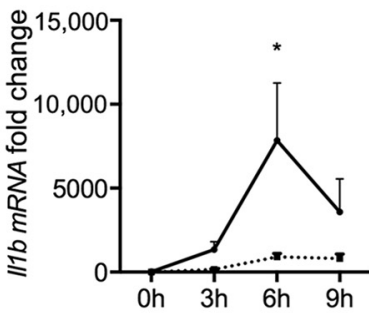

$\mathbf{E}$

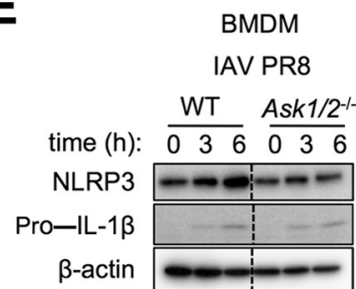

Figure 2 Apoptosis-signal regulating kinases 1/2 (ASK1/2) are required for up-regulation of Nod-like receptor family pyrin domain containing 3 (NLRP3) and

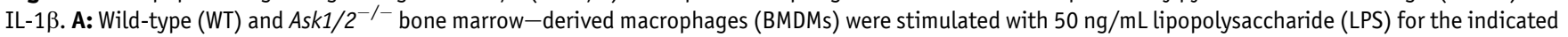
time, and NLRP3, pro-IL-1 $\beta$, and $\beta$-actin were measured by immunoblot. B: Reverse transcription-PCR analysis was used to measure the relative expression of Nlrp3 and Il $1 b$ normalized to expression of the $\beta$-actin gene after LPS stimulation. C-E: Immunoblots for NLRP3, pro-IL-1 $\beta$, and $\beta$-actin after stimulation with 50 $\mathrm{ng} / \mathrm{mL}$ LPS in primary lung fibroblasts (C), infection of BMDMs with $B$. thailandensis (multiplicity of infection, 10) (D), or infection of BMDMs with IAV PR8 (multiplicity of infection, 25) (E). $n \geq 3$ independent experiments. ${ }^{*} P<0.05$ versus WT ( $t$-test with the Holm-Sidak multiple comparisons test). IAV PR8, influenza A virus strain A/Puerto Rico/8/1934 H1N1.

(Figure 2, D and E). These data show that NLRP3 expression is regulated positively by the ASK $1 / 2$ signaling complex.

\section{ASK1/2 Are Required for NLRP3 Inflammasome-Driven Pathology during Burkholderia Infection}

To determine whether ASK1/2 regulate inflammasome activation during $B$. thailandensis infection, BMDMs from WT,

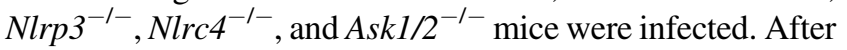
20 hours of infection, caspase- 1 cleavage was measured by immunoblot (Figure 3A), as well as lactate dehydrogenase and IL-1 $\beta$ release (Figure 3B), and a decrease in NLRP3dependent inflammasome activation and pyroptosis was observed in Askl/2 ${ }^{-1-}$ BMDMs compared with WT. To determine whether ASK1/2 contributes to $B$. thailandensis infection in vivo, WT and Askl/2-l- mice were inoculated intranasally with $5 \times 10^{5}$ colony forming units and monitored for survival. Mice lacking ASK1/2 were protected from lethal infection (Figure 3C) and had reduced bacterial numbers in the spleen at day 2 after inoculation, whereas lung colony forming units were not significantly different (Figure 3D). There were several notable quantitative and qualitative differences between WT and Askl/2 ${ }^{-1-}$ mice in both the distribution and severity of pulmonary lesions (Figure 3F). Although neutrophils and macrophages comprised the predominant inflammatory cell infiltrates in both groups, the resulting suppurative and pyogranulomatous lesions generally were larger and coalescing in WT mice, and thus affected a much higher percentage of the alveolar parenchyma than the less clearly defined neutrophil infiltrates in the $A s k 1 / 2^{-1-}$ mice (23\% versus $6 \%$, respectively) (Figure 3F). The pyogranulomatous lesions in WT mice were scattered randomly through the lung field and had more clearly defined margins than the lesions in $A s k 1 / 2^{-/}$mice. Inflammatory lesions in WT mice were characterized by a central suppurative core containing abundant degenerating neutrophils, with more epithelioid macrophages mixed with neutrophils toward the margins. In contrast, pulmonary lesions in $A s k 1 / 2^{-1-}$ mice consisted almost entirely of viable neutrophils and abundant macrophages. Other prominent lesions in WT mice that were minimal to absent in the Askl/2 ${ }^{-/-}$lungs included marked edema (both alveolar and perivascular) as well as scattered bronchioles plugged with suppurative exudates (Figure 3F). Consistent with this reduced inflammatory response, a reduction in caspase-1 cleavage (Figure 3E) and reduced proinflammatory cytokines IL- $1 \beta$, tumor necrosis factor, and IL-6 (Figure 3G) also were observed. These data suggest that proinflammatory signaling and inflammasome activation downstream of ASK1/2 is responsible for the pathology associated with intranasal $B$. thailandensis infection.

\section{ASK2 Contributes to Burkholderia-Mediated Pathology in Vivo}

The individual contribution of ASK1 and ASK2 to NLRP3 inflammasome priming and in vivo pathology was determined using BMDMs from Ask1 $1^{--}$, Ask2 $2^{--}$, and Ask $12^{-1-}$ mice. BMDMs stimulated with LPS showed that both ASK1 and ASK2 contributed partially to NLRP3 up-regulation (Figure 4A), and this lead to partial reductions in caspase- 
A

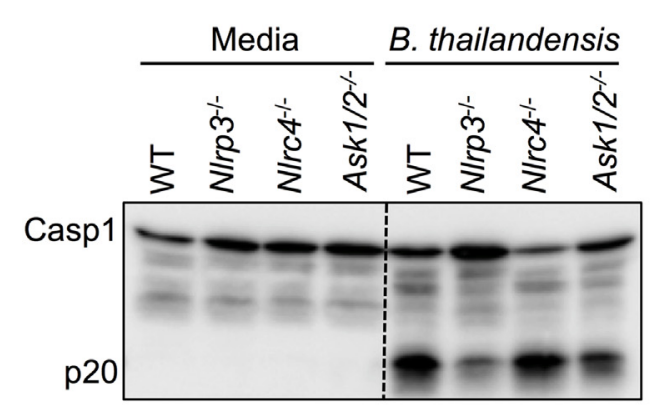

B

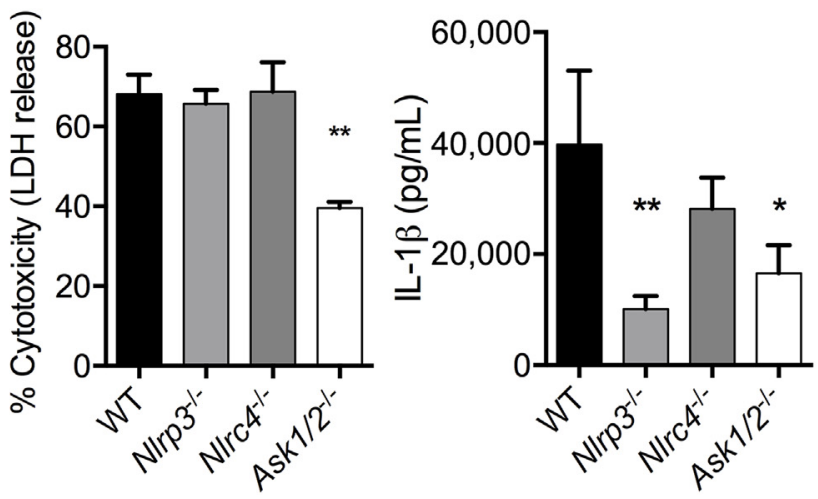

$\mathbf{E}$

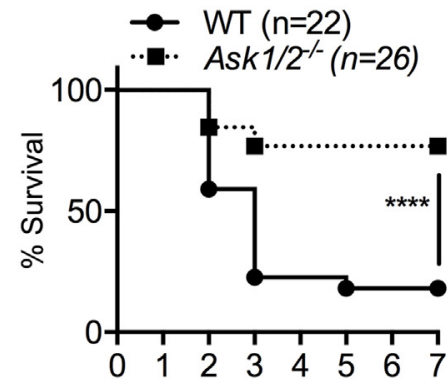

Days after inoculation

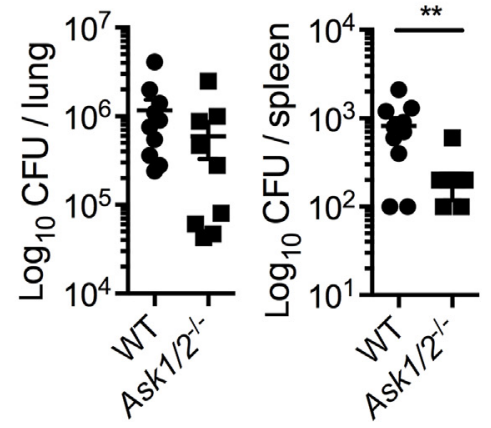

$p^{s^{t}}$

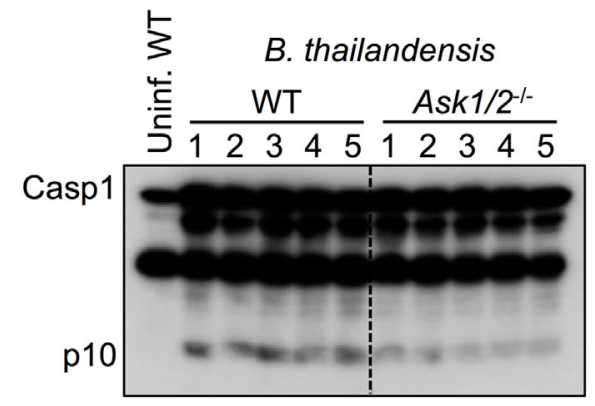

$\mathbf{F}$

G
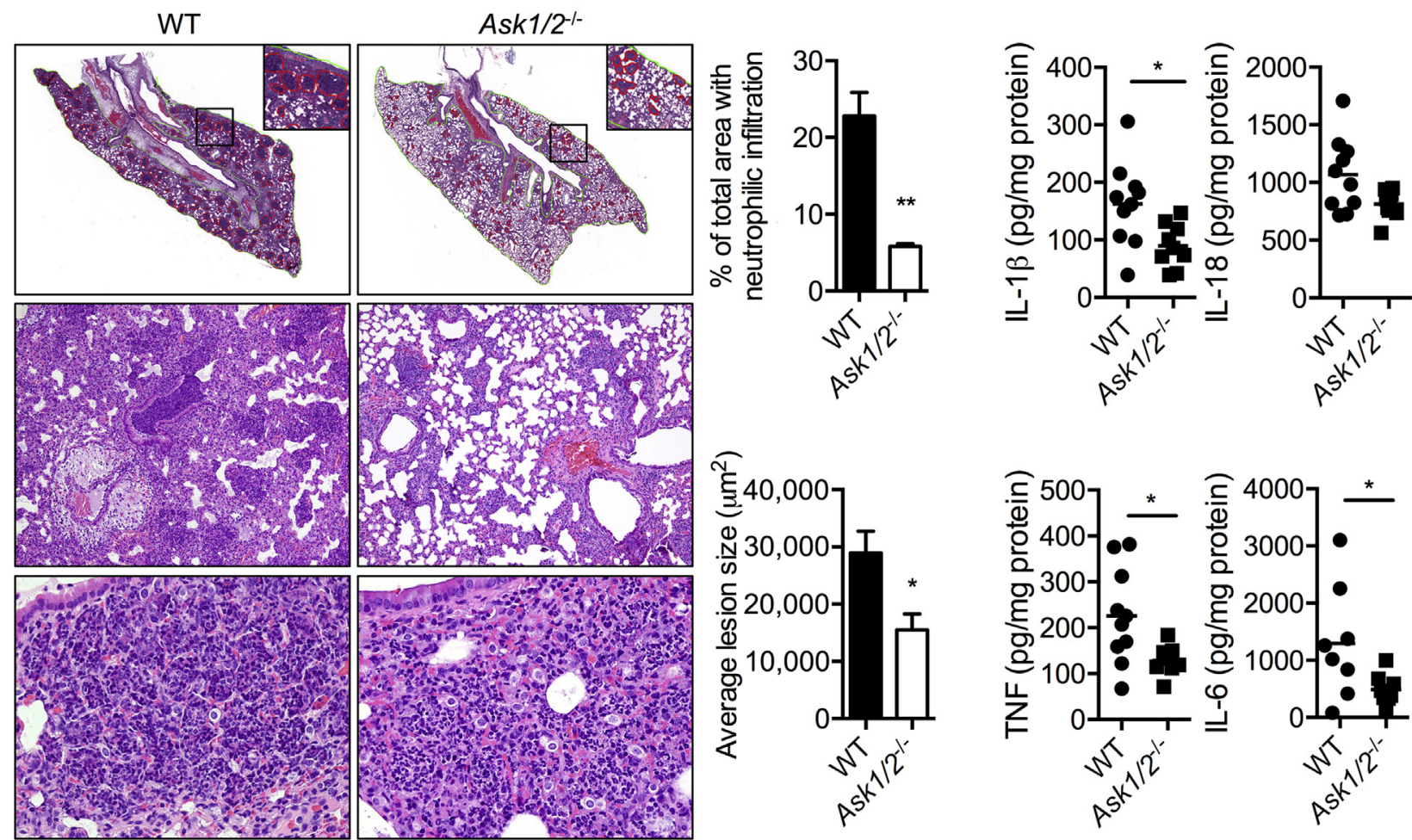
1 cleavage in single-knockout BMDMs treated with LPS and ATP (Figure 4B). Interestingly, B. thailandensis-infected BMDMs have reduced NLRP3 expression and caspase-1 cleavage in $A s k 2^{-1-}$ and $A s k 1 / 2^{-1-}$ BMDMs, but not $A s k 1^{-1-}$ cells, suggesting other TLRs may signal via ASK1/2 in distinct ways (Figure 4, C and D). Mice challenged intranasally with $B$. thailandensis are protected when ASK2 or both ASK1 and ASK2 are deleted (Figure 4E), but not when ASK1 alone is deleted. By examining baseline tissue expression from the RIKEN FANTOM5 project database, ${ }^{35}$ ASK2 was found to be very highly expressed in murine lungs compared with ASK1 (Figure 4F). Taken together, the limited role of ASK1 in BMDMs and the lower expression of ASK1 relative to ASK2 in the lung suggest that ASK2 contributes more to respiratory pathology during $B$. thailandensis infection.

\section{Discussion}

In this study, the ASK1/2 complex was found to regulate the expression of NLRP3 and downstream inflammasome activation by LPS and bacterial infection using knockout mouse models. Our results suggest that ASK2 plays a predominant role in the pathology associated with intranasal $B$. thailandensis infection independent from ASK1. Recent work by Okazaki et $\mathrm{al}^{13}$ showed that ASK1 and ASK2 have distinct functions in regulating inflammatory cytokines and cell death during influenza infection and these findings suggest other infection models will reveal unique interactions between pathogens and regulation of the ASK1/2 complex. Although most previous research focused on the role of ASK1 signaling in apoptosis, this study showed that ASK1/2 can regulate pyroptotic cell death, suggesting it may have additional functions in other cell death scenarios and in different cell types.

Much of the work on the ASK family MAP3Ks has examined the role of ASK1 in stress responses and how this leads to downstream JNK and p38 apoptotic signaling. ASK2 associates in complexes with ASK1 and each molecule regulates the other through distinct mechanisms. ${ }^{6,36}$ In the absence of ASK1, ASK2 is degraded constitutively so it is interesting that $A s k 1^{-1-}$ do not mirror the phenotypes observed in $A s k 2^{-1-}$ and $A s k 1 / 2^{-1-}$ knockout mice. ${ }^{6}$ In a study by Iriyama et al, ${ }^{11}$ ASK2 but not ASK1 limited 7,12-
dimethylbenz[a]anthracene/12-O-tetradecanoylphorbol-13acetate-induced skin tumorigenesis and also was involved in gastrointestinal cancers. The mechanism they proposed suggested that ASK2 acts as a tumor suppressor in epithelial cancer cell lines whereas ASK1 drives cytokine production.

How ASK1 and ASK2 regulate the expression of NLRP3 remains an interesting area of future study. ASK1 has been shown to phosphorylate MKK3/6 and MKK4/7 to engage p38 and JNK signaling, respectively, but downstream targets of ASK2 have not been well characterized. Interestingly, ASK2 phosphorylates ASK1 at Thr845 but ASK1 does not phosphorylate ASK2; instead, binding stabilizes ASK2 and enhances its activity in a phosphorylation-independent manner, suggesting that tissue level differences in ASK2 may regulate the activity of ASK $1 .{ }^{6}$ In addition, a third ASK family member, ASK3, also has been identified by sequence homology and interacts with ASK1, suggesting that it also may play a role in ASK1 and ASK2 signaling cascades. ${ }^{4,5}$ Adding to the complexity of these interactions, reciprocal regulation of ASK1 and transforming growth factor$\beta$-activated kinase 1 also was observed with transforming growth factor- $\beta$-activated kinase 1 during Helicobacter pylori infection and after stimulation with $\mathrm{H}_{2} \mathrm{O}_{2}$ or tumor necrosis factor-induced apoptosis, suggesting these complexes may regulate NF- $\mathrm{B}$ or AP-1-dependent up-regulation of NLRP3. ${ }^{37,38}$ It will be interesting to explore whether ASK2 regulates the activity of other MAP3Ks and downstream targets to regulate NLRP3 expression.

In this study, the high mortality of WT mice in comparison with $A s k 1 / 2^{-\prime-}$ mice is most likely owing to the more severe histopathologic lesions in the lungs of WT mice, which reduced the proportion of remaining functional alveoli. It is likely that the increased degeneration and necrosis of neutrophils in the pulmonary lesions of WT mice contributed to the increased influx of inflammatory cell infiltrates, as well as the more extensive pulmonary edema and numerous exudate-plugged airways. In contrast, the neutrophilic infiltrates in Askl/2 ${ }^{-/-}$mice were milder and consisted mostly of viable neutrophils, which would be consistent with a decrease in pyroptosis. The milder infiltrates of viable neutrophils in Ask1/2 ${ }^{--}$mice were associated with minimal pulmonary edema and bronchiolar plugging, and thus retention of functional peripheral alveoli that were mostly unaffected by inflammatory cell infiltrates or exudates.

Figure 3 Apoptosis-signal regulating kinases 1/2 (ASK1/2) are required for Nod-like receptor family pyrin domain containing 3 (NLRP3) inflammasomedriven pathology during Burkholderia infection. A: Immunoblot for caspase-1 cleavage after bone marrow-derived macrophage (BMDM) infection with $B$. thailandensis (multiplicity of infection, 10; 20 hours). B: Release of lactate dehydrogenase (LDH) and IL-1 $\beta$ in the supernatants of BMDMs infected with $B$. thailandensis. C: Survival curve from wild-type (WT) and $A$ sk1 $12^{-/-}$mice infected intranasally with $5 \times 10^{5}$ colony forming units (CFU) of $B$. thailandensis. D: $B$. thailandensis CFU recovered from homogenized lung or spleen at day 2 after inoculation. E: Immunoblot for caspase-1 p10 cleavage in normalized lung homogenates from day 2 after infection. F: Hematoxylin and eosin staining of lungs fixed in formalin at day 2 of infection with $B$. thailandensis, with green lines indicating total area and red outlines indicating the lesion area used for quantitative analysis. Boxed areas are shown at higher magnification in the insets. Insets magnify the image analysis strategy to measure lesion size and total lung area. G: Cytokine enzyme-linked immunosorbent assay from normalized lung homogenate supernatants at day 2 after infection. Data are representative from at least three independent experiments, with significance determined by one-way analysis of variance compared with WT with the Dunn multiple comparison test (B), by the log-rank test (C), or by the Mann-Whitney test $(\mathbf{D}, \mathbf{F}$, and $\mathbf{G}) .{ }^{*} P<0.05,{ }^{*} P<0.005$, and ${ }^{* * * *} P<0.00005$ versus WT. Original magnification: $\times 2(\mathbf{F}$, top row $) ; \times 10(\mathbf{F}$, middle row); $\times 40(\mathbf{F}$, bottom row); $\times 4$ (F, inset). TNF, tumor necrosis factor; Uninf, uninfected. 
A

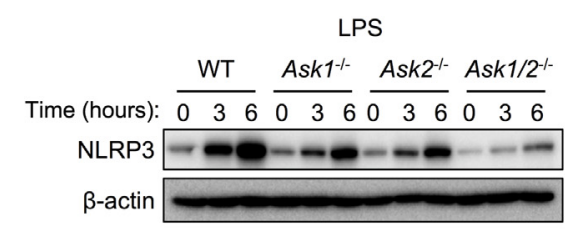

C

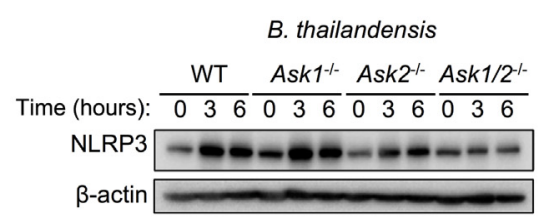

E

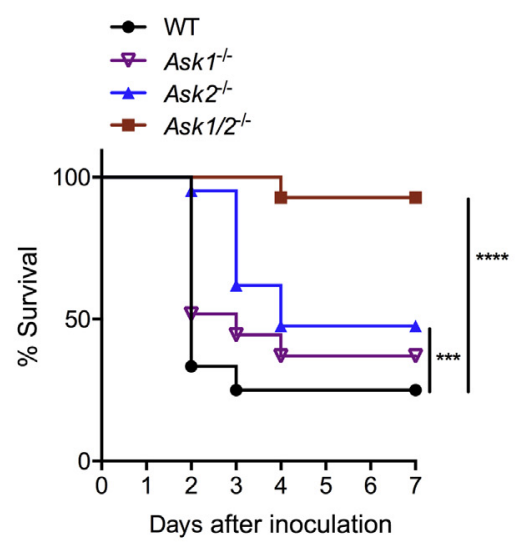

B

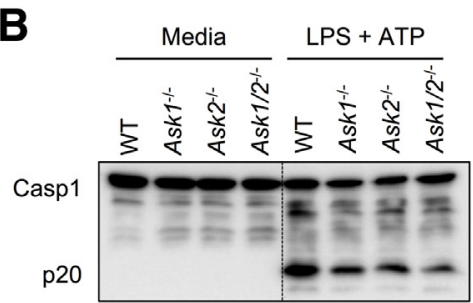

D

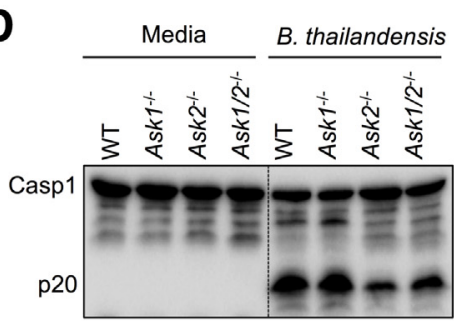

$\mathbf{F}$

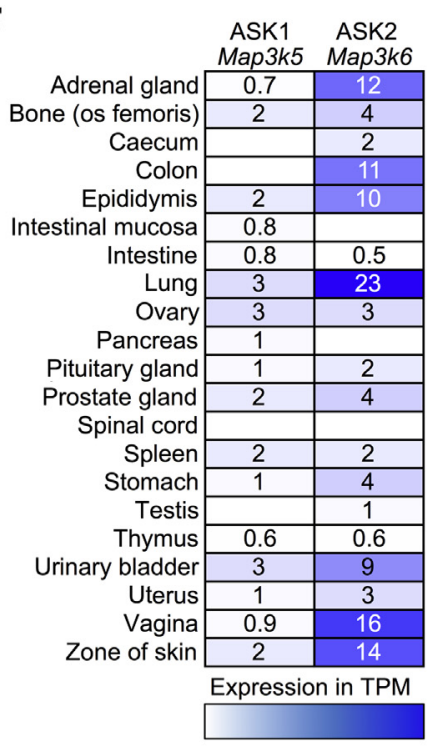

Figure 4 Apoptosis-signal regulating kinase 1 (ASK1) and ASK2 regulate Nod-like receptor family pyrin domain containing 3 (NLRP3) inflammasome priming but ASK2 predominantly contributes during intranasal infection. A: Bone marrow-derived macrophages (BMDMs) from wild-type (WT) or knockout mice were stimulated with $50 \mathrm{ng} / \mathrm{mL}$ lipopolysaccharide (LPS) and expression of NLRP3 and $\beta$-actin were measured by immunoblot at the indicated time points. B: Caspase- 1 cleavage was measured after stimulation with LPS and ATP. BMDMs from WT or knockout mice were stimulated with $B$. thailandensis (multiplicity of infection, 10) and expression of NLRP3 and $\beta$-actin were measured by immunoblot at the indicated time points (C) and caspase-1 cleavage was measured (D). E: Survival curves were generated after intranasal infection of mice with $5 \times 10^{5}$ CFU $B$. thailandensis. F: Tissue expression of ASK1 (Map3k5) and ASK2 (Map3k6) was generated using publicly available data from the RIKEN FANTOM5 project database (http://fantom.gsc.riken.jp/5) and EMBL-EBI Expression Atlas (http://www.ebi. ac.uk/gxa/home) with expression in transcripts per million (TPM). E: Data are representative from at least three independent experiments or pooled, with significance determined by the logrank test. $n=24$ WT (E); $n=27 A s k 1^{-/-}(\mathbf{E})$; $n=21 \mathrm{Ask2}^{-/-}(\mathrm{E}) ; n=14 \mathrm{Ask} 1 / 2^{-/-}(\mathrm{E})$. $* * * P<0.0005, * * * * P<0.00005$.
The initial route of inoculation strongly influences the distribution of pulmonary lesions, and intranasal inoculations of pulmonary pathogens generally results in centripetal spread of infection from proximal airways and central bronchi to the lung periphery. However, the diffuse random distribution of pulmonary lesions that was observed in B. thailandensis infected mice typically is associated with a hematogenous route of infection. Taken together, these findings suggest that mice inoculated intranasally with B. thailandensis develop an early bacteremia with hematogenous spread to the lung.

Comparing the responses to the diverse stimuli that lead to ASK1/2 and NLRP3-dependent cell death, some common themes emerged. Each pathway was activated downstream of TLR4, required the activity of reactive oxygen species, and each was associated with regulatory proteins including thioredoxin interacting protein, heat shock protein 90 , and A20. ${ }^{8,39-44}$ Interestingly, receptor interacting serine/threonine kinase 1 , which regulates apoptotic, necroptotic, and some pyroptotic pathways, has been shown to regulate ASK1 activity downstream of tumor necrosis factor, suggesting it may link these pathways. ${ }^{39,45,46}$ Understanding where these pathways interact represents potential new targets for regulating inflammatory diseases. Specific inhibitors of ASK1 or ASK2 may be useful for fine-tuning cellular stress responses and limiting overt pathologic inflammation or cell death.

In summary, our data show that the ASK1/2 signaling complex regulates NLRP3 expression and subsequent NLRP3 inflammasome activation downstream of TLR4 and during infection by $B$. thailandensis. The deletion of ASK2 but not ASK1 specifically contributes to limiting inflammatory pathology in the lungs of mice infected with B. thailandensis, and ASK1/2 signaling is associated with reduced inflammation, caspase-1 cleavage, and proinflammatory cytokines in the lung. These findings suggest that ASK family kinases may regulate other NLRP3dependent cell processes in response to different stimuli or in different cellular contexts, contributing to inflammatory disease and cell death processes beyond apoptosis. 


\section{Acknowledgment}

We thank Dr. Joseph Mougous (University of Washington) for providing the Burkholderia strain used in this study.

\section{Supplemental Data}

Supplemental material for this article can be found at https://doi.org/10.1016/j.ajpath.2017.12.006.

\section{References}

1. Cargnello M, Roux PP: Activation and function of the MAPKs and their substrates, the MAPK-activated protein kinases. Microbiol Mol Biol Rev 2011, 75:50-83

2. Arthur JSC, Ley SC: Mitogen-activated protein kinases in innate immunity. Nat Rev Immunol 2013, 13:679-692

3. Ajibade AA, Wang Q, Cui J, Zou J, Xia X, Wang M, Tong Y, Hui W, Liu D, Su B, Wang HY, Wang R-F: TAK1 negatively regulates NF-кB and p38 MAP kinase activation in $\mathrm{Gr}-1+\mathrm{CD} 11 \mathrm{~b}+$ neutrophils. Immunity 2012, 36:43-54

4. Kaji T, Yoshida S, Kawai K, Fuchigami Y, Watanabe W, Kubodera H, Kishimoto T: ASK3, a novel member of the apoptosis signalregulating kinase family, is essential for stress-induced cell death in HeLa cells. Biochem Biophys Res Commun 2010, 395:213-218

5. Federspiel JD, Codreanu SG, Palubinsky AM, Winland AJ, Betanzos CM, McLaughlin B, Liebler DC: Assembly dynamics and stoichiometry of the apoptosis signal-regulating kinase (ASK) signalosome in response to electrophile stress. Mol Cell Proteomics 2016, 15:1947-1961

6. Takeda K, Shimozono R, Noguchi T, Umeda T, Morimoto Y, Naguro I, Tobiume K, Saitoh M, Matsuzawa A, Ichijo H: Apoptosis signal-regulating kinase (ASK) 2 functions as a mitogen-activated protein kinase kinase kinase in a heteromeric complex with ASK1. J Biol Chem 2007, 282:7522-7531

7. Tobiume K, Matsuzawa A, Takahashi T, Nishitoh H, Morita K, Takeda K, Minowa O, Miyazono K, Noda T, Ichijo H: ASK1 is required for sustained activations of JNK/p38 MAP kinases and apoptosis. EMBO Rep 2001, 2:222-228

8. Zhang L, Chen J, Fu H: Suppression of apoptosis signal-regulating kinase 1-induced cell death by 14-3-3 proteins. Proc Natl Acad Sci U S A 1999, 96:8511-8515

9. Fujino G, Noguchi T, Matsuzawa A, Yamauchi S, Saitoh M, Takeda K, Ichijo H: Thioredoxin and TRAF family proteins regulate reactive oxygen species-dependent activation of ASK1 through reciprocal modulation of the N-terminal homophilic interaction of ASK1. Mol Cell Biol 2007, 27:8152-8163

10. Hatai T, Matsuzawa A, Inoshita S, Mochida Y, Kuroda T, Sakamaki K, Kuida K, Yonehara S, Ichijo H, Takeda K: Execution of apoptosis signalregulating kinase 1 (ASK1)-induced apoptosis by the mitochondriadependent caspase activation. J Biol Chem 2000, 275:26576-26581

11. Iriyama T, Takeda K, Nakamura H, Morimoto Y, Kuroiwa T, Mizukami J, Umeda T, Noguchi T, Naguro I, Nishitoh H, Saegusa K, Tobiume K, Homma T, Shimada Y, Tsuda H, Aiko S, Imoto I, Inazawa J, Chida K, Kamei Y, Kozuma S, Taketani Y, Matsuzawa A, Ichijo H: ASK1 and ASK2 differentially regulate the counteracting roles of apoptosis and inflammation in tumorigenesis. EMBO J 2009, 28:843-853

12. Maruoka S, Hashimoto S, Gon Y, Nishitoh H, Takeshita I, Asai Y, Mizumura K, Shimizu K, Ichijo H, Horie T: ASK1 regulates influenza virus infection-induced apoptotic cell death. Biochem Biophys Res Commun 2003, 307:870-876

13. Okazaki T, Higuchi M, Takeda K, Iwatsuki-Horimoto K, Kiso M, Miyagishi M, Yanai H, Kato A, Yoneyama M, Fujita T, Taniguchi T, Kawaoka Y, Ichijo H, Gotoh Y: The ASK family kinases differentially mediate induction of type I interferon and apoptosis during the antiviral response. Sci Signal 2015, 8:ra78

14. Matsuzawa A, Saegusa K, Noguchi T, Sadamitsu C, Nishitoh H, Nagai S, Koyasu S, Matsumoto K, Takeda K, Ichijo H: ROS-dependent activation of the TRAF6-ASK1-p38 pathway is selectively required for TLR4-mediated innate immunity. Nat Immunol 2005, 6:587-592

15. Martinon F, Burns K, Tschopp J: The inflammasome: a molecular platform triggering activation of inflammatory caspases and processing of proIL-beta. Mol Cell 2002, 10:417-426

16. Kesavardhana S, Kanneganti TD: Mechanisms governing inflammasome activation, assembly and pyroptosis induction. Int Immunol 2017, 29:201-210

17. Ceballos-Olvera I, Sahoo M, Miller MA, Del Barrio L, Re F: Inflammasome-dependent pyroptosis and IL-18 protect against Burkholderia pseudomallei lung infection while IL-1 $\beta$ is deleterious. PLoS Pathog 2011, 7:e1002452

18. Man SM, Karki R, Kanneganti T-D: Molecular mechanisms and functions of pyroptosis, inflammatory caspases and inflammasomes in infectious diseases. Immunol Rev 2017, 277:61-75

19. Man SM, Karki R, Malireddi RKS, Neale G, Vogel P, Yamamoto M, Lamkanfi M, Kanneganti T-D: The transcription factor IRF1 and guanylate-binding proteins target activation of the AIM2 inflammasome by Francisella infection. Nat Immunol 2015, 16:467-475

20. Tzeng TC, Schattgen S, Monks B, Wang D, Cerny A, Latz E, Fitzgerald K, Golenbock DT: A fluorescent reporter mouse for inflammasome assembly demonstrates an important role for cell-bound and free ASC specks during in vivo infection. Cell Rep 2016, 16:571-582

21. Soga M, Matsuzawa A, Ichijo H: Oxidative stress-induced diseases via the ASK1 signaling pathway. Int J Cell Biol 2012, 2012:439587

22. Perregaux D, Gabel CA: Interleukin-1 beta maturation and release in response to ATP and nigericin. Evidence that potassium depletion mediated by these agents is a necessary and common feature of their activity. J Biol Chem 1994, 269:15195-15203

23. Agostini L, Martinon F, Burns K, McDermott MF, Hawkins PN, Tschopp J: NALP3 forms an IL-1beta-processing inflammasome with increased activity in Muckle-Wells autoinflammatory disorder. Immunity 2004, 20:319-325

24. Zhao Y, Shao F: The NAIP-NLRC4 inflammasome in innate immune detection of bacterial flagellin and type III secretion apparatus. Immunol Rev 2015, 265:85-102

25. Bürckstümmer T, Baumann C, Blüml S, Dixit E, Dürnberger G, Jahn H, Planyavsky M, Bilban M, Colinge J, Bennett KL, Superti-Furga G: An orthogonal proteomic-genomic screen identifies AIM2 as a cytoplasmic DNA sensor for the inflammasome. Nat Immunol 2009, 10:266-272

26. Hornung V, Ablasser A, Charrel-Dennis M, Bauernfeind F, Horvath G, Caffrey DR, Latz E, Fitzgerald KA: AIM2 recognizes cytosolic dsDNA and forms a caspase-1-activating inflammasome with ASC. Nature 2009, 458:514-518

27. Roberts TL, Idris A, Dunn JA, Kelly GM, Burnton CM, Hodgson S, Hardy LL, Garceau V, Sweet MJ, Ross IL, Hume DA, Stacey KJ: HIN-200 proteins regulate caspase activation in response to foreign cytoplasmic DNA. Science 2009, 323:1057-1060

28. Fernandes-Alnemri T, Yu J-W, Datta P, Wu J, Alnemri ES: AIM2 activates the inflammasome and cell death in response to cytoplasmic DNA. Nature 2009, 458:509-513

29. He W-T, Wan H, Hu L, Chen P, Wang X, Huang Z, Yang ZH, Zhong CQ, Han J: Gasdermin D is an executor of pyroptosis and required for interleukin-1 $\beta$ secretion. Cell Res 2015, 25:1285-1298

30. Kayagaki N, Stowe IB, Lee BL, O'Rourke K, Anderson K, Warming S, Cuellar T, Haley B, Roose-Girma M, Phung QT, Liu PS, Lill JR, Li H, Wu J, Kummerfeld S, Zhang J, Lee WP, Snipas SJ, Salvesen GS, Morris LX, Fitzgerald L, Zhang Y, Bertram EM, Goodnow CC, Dixit VM: Caspase-11 cleaves gasdermin D for noncanonical inflammasome signalling. Nature 2015, 526:666-671

31. Choi J-R, Heo H, Lang Y, Shin KS, Kang SJ: Apoptosis signalregulating kinase 1 regulates the expression of caspase-11. FEBS Lett 2009, 583:3016-3020 
32. Gurung P, Malireddi RKS, Anand PK, Demon D, Walle LV, Liu Z, Vogel P, Lamkanfi M, Kanneganti TD: TRIF-mediated caspase-11 production integrates TLR4- and Nlrp3 inflammasome-mediated host defense against enteropathogens. J Biol Chem 2012, 287:34474-34483

33. Rathinam VAK, Vanaja SK, Waggoner L, Sokolovska A, Becker C, Stuart LM, Leong JM, Fitzgerald KA: TRIF licenses caspase-11dependent NLRP3 inflammasome activation by gram-negative bacteria. Cell 2012, 150:606-619

34. Sharma D, Kanneganti T-D: The cell biology of inflammasomes: mechanisms of inflammasome activation and regulation. J Cell Biol 2016, 213:617-629

35. Lizio M, Harshbarger J, Shimoji H, Severin J, Kasukawa T, Sahin S, et al: Gateways to the FANTOM5 promoter level mammalian expression atlas. Genome Biol 2015, 16:22

36. Cockrell LM, Puckett MC, Goldman EH, Khuri FR, Fu H: Dual engagement of 14-3-3 proteins controls signal relay from ASK2 to the ASK1 signalosome. Oncogene 2009, 29:822-830

37. Hayakawa Y, Hirata Y, Kinoshita H, Sakitani K, Nakagawa H, Nakata W, Takahashi R, Sakamoto K, Maeda S, Koike K: Differential roles of ASK1 and TAK1 in Helicobacter pylori-induced cellular responses. Infect Immun 2013, 81:4551-4560

38. Kim SY, Shim J-H, Chun E, Lee KY: Reciprocal inhibition between the transforming growth factor- $\beta$-activated kinase 1 (TAK1) and apoptosis signal-regulating kinase 1 (ASK1) mitogen-activated protein kinase kinase kinases and its suppression by TAK1-binding protein 2 (TAB2), an adapter protein for TAK1. J Biol Chem 2012, 287:3381-3391

39. Zhang H, Zhang R, Zhang H, Lin Y, Li J, Pober JS, Min W: RIP1mediated AIP1 phosphorylation at a 14-3-3-binding site is critical for tumor necrosis factor-induced ASK1-JNK/p38 activation. J Biol Chem 2007, 282:14788-14796
40. Won M, Park KA, Byun HS, Sohn K-C, Kim Y-R, Jeon J, Hong JH, Park J, Seok JH, Kim JM, Yoon W-H, Jang I-S, Shen HM, Liu ZG, Hur GM: Novel anti-apoptotic mechanism of A20 through targeting ASK1 to suppress TNF-induced JNK activation. Cell Death Differ 2010, 17:1830-1841

41. Vande Walle L, Van Opdenbosch N, Jacques P, Fossoul A, Verheugen E, Vogel P, Beyaert R, Elewaut D, Kanneganti T-D, van Loo G, Lamkanfi M: Negative regulation of the NLRP3 inflammasome by A20 protects against arthritis. Nature 2014, 512:69-73

42. Zhou R, Tardivel A, Thorens B, Choi I, Tschopp J: Thioredoxininteracting protein links oxidative stress to inflammasome activation. Nat Immunol 2010, 11:136-140

43. Mayor A, Martinon F, De Smedt T, Pétrilli V, Tschopp J: A crucial function of SGT1 and HSP90 in inflammasome activity links mammalian and plant innate immune responses. Nat Immunol 2007, 8: 497-503

44. Zhang R, Luo D, Miao R, Bai L, Ge Q, Sessa WC, Min W: Hsp90-Akt phosphorylates ASK1 and inhibits ASK1-mediated apoptosis. Oncogene 2005, 24:3954-3963

45. Kuriakose T, Man SM, Malireddi RKS, Karki R, Kesavardhana S, Place DE, Neale G, Vogel P, Kanneganti T-D: ZBP1/DAI is an innate sensor of influenza virus triggering the NLRP3 inflammasome and programmed cell death pathways. Sci Immunol 2016, 1:aag2045

46. Nogusa S, Thapa RJ, Dillon CP, Liedmann S, Oguin TH III, Ingram JP, Rodriguez DA, Kosoff R, Sharma S, Sturm O, Verbist K, Gough PJ, Bertin J, Hartmann BM, Sealfon SC, Kaiser WJ, Mocarski ES, López CB, Thomas PG, Oberst A, Green DR, Balachandran S: RIPK3 activates parallel pathways of MLKL-driven necroptosis and FADD-mediated apoptosis to protect against influenza A virus. Cell Host Microbe 2016, 20:13-24 